\title{
Simple Solutions to Steady-State Cumulus Regimes in the Convective Boundary Layer
}

\author{
JERÔME SCHALKWIJK AND HARMEN J. J. JONKER \\ Delft University of Technology, Delft, Netherlands
}

\section{A. Pier Siebesma}

Delft University of Technology, Delft, and Royal Netherlands Meteorological Institute, De Bilt, Netherlands

(Manuscript received 16 November 2012, in final form 17 June 2013)

\begin{abstract}
A modeling framework is developed that extends the mixed-layer model to steady-state cumulus convection. The aim is to consider the simplest model that retains the essential behavior of cumulus-capped layers. The presented framework allows for the evaluation of stationary states dependent on external parameters. These states are completely independent of the initial conditions, and therefore represent an asymptote that might help deepen understanding of the dependence of the cloudy boundary layer on external forcings. Formulating separate equations for the lifting condensation level and the mixed-layer height, the dry and wet energetics can be distinguished. Regimes that can support steady-state cumulus clouds and regimes that cannot are identified by comparison of the dry and wet buoyancy effects. The dominant mechanisms that govern the creation and eventual depth of the cloud layer are identified. Model predictions are tested by comparison with a large number of independent large-eddy simulations for varying surface and large-scale conditions and are found to be in good agreement.
\end{abstract}

\section{Introduction}

Boundary layer clouds play an important role in both the dynamical and radiative properties of the boundary layer, controlling to an important extent the height, the efficiency of vertical transport, and the opacity of the boundary layer. However, these clouds are also notoriously difficult to model owing to the high resolution needed to resolve boundary layer turbulence. Stratocumulus cloud decks require high vertical resolution to resolve cloud-top entrainment, and properties of cumulus clouds are also sensitive to the horizontal grid resolution because of their inhomogeneity.

Understanding of cumulus clouds has increased significantly over the past decades, in part because of largeeddy simulations (LESs; e.g., Deardorff 1970), which allowed many numerical studies to be performed on detailed cloud processes. Specifically, understanding the cloud-environment interactions (Paluch 1979; Reuter and Yau 1987; Siebesma and Cuijpers 1995; Heus and

Corresponding author address: Jerôme Schalkwijk, Delft University of Technology, Stevinweg 1, 2628 CN Delft, Netherlands. E-mail: j.schalkwijk@tudelft.nl
Jonker 2008) has been a popular topic over many years, enhancing our understanding of cumulus clouds and improving parameterizations. However, the behavior of the cumulus-capped boundary layer as a whole and the interplay with large-scale tendencies and forcings is still poorly understood. This is further emphasized by studies on the wide spread of cloud-climate feedbacks (Dufresne and Bony 2008; Bony et al. 2006), revealing the large uncertainties associated with the feedback behavior of low clouds.

For this reason, this work attempts a modeling approach as simple as possible while retaining the essential behavior. Starting with the mixed-layer model for clearconvective situations, building on the work of Tennekes (1973), Lilly (1968), and, more recently, Vilà-Guerau de Arellano et al. (2004) and van Driel and Jonker (2011), the model is further elaborated to include a cloud layer, working in the line of Stevens (2006), Neggers et al. (2006), Stevens (2007), Bellon and Stevens (2012), and Bellon and Stevens (2013). The goal of this exercise is to set up a framework in which one can study the response of cumuliform clouds to large-scale atmospheric forcings and surface properties, minimizing complications by simplifying the system to its essence. Note that in 
doing this, a somewhat different approach is chosen than, for example, Bretherton and Park (2008) and Nuijens and Stevens (2012), who propose more realistic models that focus on the dynamical temporal response of the cloud layer, whereas we focus on the stationary solutions.

The focus on stationary solutions allows us to formulate analytical solutions for the regimes where cumuliform clouds are supported. These solutions can be solved analytically or numerically but do not require model integration in time. The hope is that such solutions further promote our understanding of the limiting factors on cumulus regimes. Moreover, these solutions can be used to quickly set up numerical experiments of steady-state cumulus convection in varying environmental conditions and forcings, which might aid future steady-state cumulus studies.

\section{Bulk modeling}

Consider a conserved scalar $\psi$. The evolution of the ensemble average $\bar{\psi}$ is described by the following conservation equation (e.g., Stevens 2006):

$$
\frac{\partial \bar{\psi}}{\partial t}+\bar{w} \frac{\partial \bar{\psi}}{\partial z}=-\frac{\partial \phi_{\psi}}{\partial z}+S_{\psi}
$$

assuming incompressibility. In this equation, $\phi_{\psi}$ denotes the vertical turbulent flux of $\psi$ (i.e., $\phi_{\psi}=\overline{w^{\prime} \psi^{\prime}}$ ), where primes indicate deviations from the ensemble mean. Sources, including radiative cooling and horizontal advection, are contained in the source term $S_{\psi}$, which can depend on height. The ensemble-mean vertical velocity $\bar{w}(z)$ is associated with large-scale motions.

In this manuscript we will focus on the steady-state solutions, allowing us to reduce Eq. (1) to

$$
\bar{w} \frac{\partial \bar{\psi}}{\partial z}=-\frac{\partial \phi_{\psi}}{\partial z}+S_{\psi}
$$

Now we assume the boundary layer to be described by profiles as depicted in Fig. 1. The profiles are assumed to be well mixed throughout the mixed layer. The mixed layer reaches up to level $\mathcal{L}$ - the lifting condensation level (LCL) - which marks the start of the cloud layer. The boundary layer top is denoted as $h$, and we assume turbulent fluxes to vanish at this level. The cloud layer is thus included in $h$. In the event that no clouds form, the profile is well mixed all the way up to the boundary layer height $h$. Above $h, \bar{\psi}$ is described by the free-tropospheric state $\bar{\psi}^{f}$.

Boundary conditions are provided by the free troposphere above the boundary layer height and the surface value $\psi_{0}$ in an infinitesimally thin surface layer. We integrate over this profile from $z=0$ to $z=h^{+}$, where we
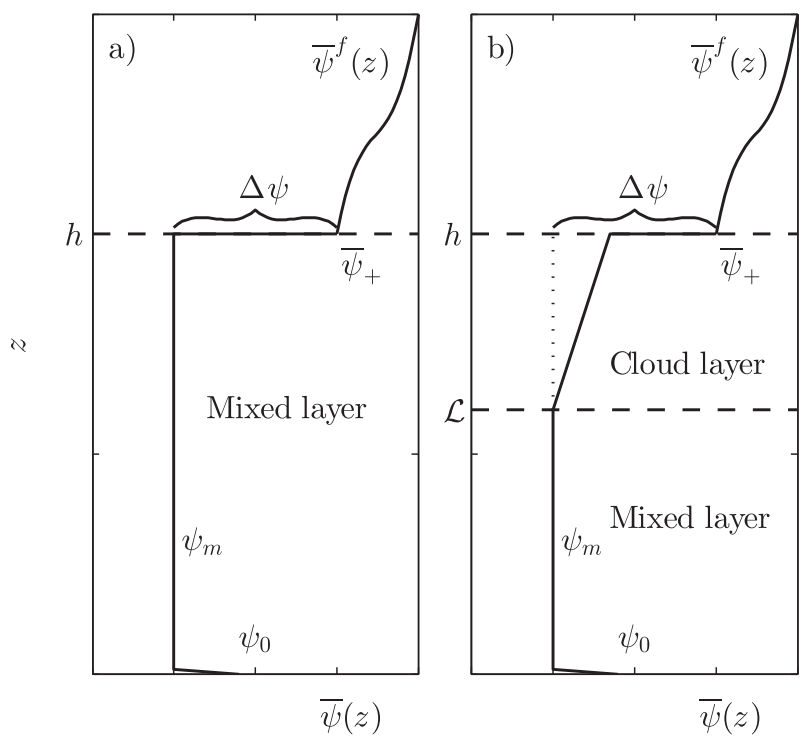

FIG. 1. Assumed profile of a conserved scalar $\psi$ for the case of (a) a clear boundary layer and (b) cumulus convection. In this framework, the "jump" is considered the difference between the value above the boundary layer (plus sign) and the mixed-layer value. Note that the profile for cumulus convection collapses to the clear boundary layer when $\mathcal{L}>h$.

define $h^{+}=\lim _{\epsilon \downarrow 0} h+\epsilon$, just above boundary layer top, to incorporate the discontinuity at the top into the integration.

Using the mixed-layer assumption that $\bar{\psi}(z)=\psi_{m}$ for $0<z<\min (\mathcal{L}, h)$, the integration yields

$$
\begin{gathered}
\bar{w}(h)\left(\bar{\psi}_{+}-\psi_{m}\right)-\int_{\mathcal{L}}^{h^{+}}\left[\bar{\psi}(z)-\psi_{m}\right] \frac{\partial \bar{w}(z)}{\partial z} d z \\
=\phi_{\psi, 0}+\int_{0}^{h^{+}} S_{\psi} d z
\end{gathered}
$$

where the turbulent flux at $h^{+}$vanishes. Subscripts are used as shorthand notation for the evaluation point; that is, $\phi_{\psi, 0}$ represents the surface flux and $\bar{\psi}_{+}$is short for $\bar{\psi}\left(h^{+}\right)=\bar{\psi}^{f}(h)$.

The effects of an eventual cloud-layer gradient enter the equation through the second term on the left-hand side. In a recent study, Bellon and Stevens (2012) attempted to capture these cloud-layer effects in the parameter $\gamma$, which can be reformulated in the current framework as follows:

$$
\gamma=1-\frac{1}{\bar{w}(h)} \int_{\mathcal{L}}^{h^{+}} \frac{\bar{\psi}(z)-\psi_{m}}{\bar{\psi}_{+}-\psi_{m}} \frac{\partial \bar{w}(z)}{\partial z} d z,
$$

which captures the effect of the interaction between the large-scale vertical velocity $\bar{w}$ and the cloud-layer 
gradient in a single dimensionless quantity. Using Eq. (4), one can rewrite Eq. (3) as

$$
\gamma \bar{w}(h)\left(\bar{\psi}_{+}-\psi_{m}\right)=\phi_{\psi, 0}+\int_{0}^{h^{+}} S_{\psi} d z .
$$

Bellon and Stevens (2012) simplified Eq. (5) by assuming $\gamma$ to have a constant value of 0.8 . To simplify even further, in this study we ignore the cloud-layer gradient, which boils down to setting $\gamma=1$; the sensitivity of the results to $\gamma$ will be evaluated in section $4 \mathrm{c}$.

\section{a. Boundary layer height}

To determine the steady-state $h$, we consider the boundary layer energetics. We follow the approach of Stevens (2007) and use what is sometimes called the "liquid water virtual potential temperature" (Grenier and Bretherton 2001):

$$
\theta_{v l}=\theta_{l}+\epsilon_{I} \theta q_{t},
$$

with $\epsilon_{I}=R_{v} / R_{d}-1$. In the absence of liquid water, $\theta_{v l}$ is equal to the virtual potential temperature. As the temperature variation in the second term is typically negligible, hereafter the liquid water virtual potential temperature is approximated as a linear combination of $\theta_{l}$ and $q_{t}$; that is, $\widetilde{\epsilon}_{I} \equiv \epsilon_{I} \theta \approx$ constant.

The result is that, to good approximation, $\theta_{v l}$ is conserved, implying that Eq. (1) also holds for $\theta_{v l}$. In the mixed layer, $\partial \theta_{v l} / \partial z=0$ and Eq. (2) for $\theta_{v l}$ reduces to

$$
\frac{\partial \phi_{\theta_{v l}}(z)}{\partial z}=S_{\theta_{v l}}(z) \text { for } \quad z<\min (\mathcal{L}, h) .
$$

In steady state, the flux divergence is required to balance the sources. If $\phi_{\theta_{v l}, 0}$ is known, Eq. (7) can be integrated to determine $\phi_{\theta_{v l}}(z)$ throughout the mixed layer.

This allows us to define the "dry thermal reach" $\eta$, which describes the height that convection would reach were there no effects from condensation (i.e., "dry" in the sense that no liquid water effects are taken into account). We define $\eta$ as the height at which

$$
\phi_{\theta_{v l}}\left(\eta^{-}\right)=-a \phi_{\theta_{v l}}(0)
$$

where $a$ is a constant fraction, representing the "entrainment efficiency." Equation (8) describes one of the fundamental properties of buoyancy-driven boundary layers (Ball 1960; Betts 1973): the energy provided by the surface buoyancy flux dissipates during the upward motion in such a way that we find the top of the cloudless boundary layer at $h=\eta$ in Eq. (8). Hence, Eq. (8) represents the classical mixed-layer closure in case no clouds form, with $\eta=h$. In case clouds form, $\eta$ becomes a maximum bound on the mixed-layer height, as we must have $\mathcal{L}<\eta$ for clouds to form.

The value of $\eta$ can be solved from combining Eqs. (7) and (8), which provides an implicit equation for $\eta$ :

$$
-\phi_{\theta_{v l}, 0}(1+a)=\int_{0}^{\eta^{-}} S_{\theta_{v l}}(z) d z .
$$

Note that it is $\eta$ that provides the criterion for cumulus cloud formation: clouds will form if the lifting condensation level is found below $\eta$ (i.e., for $\mathcal{L} \leq \eta$ ), whereas the boundary layer remains cloudless when $\mathcal{L}>\eta$. The lifting condensation level is determined by the mixedlayer values of $\theta_{l, m}$ and $q_{t, m}$, as well as the surface pressure $p_{s}$,

$$
\mathcal{L}=f\left(\theta_{l, m}, q_{t, m}, p_{s}\right)
$$

If $\mathcal{L}>\eta$, the steady-state solution describing the boundary layer state is found from Eqs. (5) and (9) with $\psi=\theta_{v l}$ and $h=\eta$.

\section{b. Cloud layer}

In general, Eq. (5) conveys that $\theta_{l, m}$ and $q_{t, m}$ will be a function of $h$. From Eq. (10), then so is the lifting condensation level: $\mathcal{L}=\mathcal{L}(h)$. Hence, a cloudless solution is possible if $\mathcal{L}(\eta)>\eta$; that is, the thermodynamic properties of a boundary layer with height $h=\eta$ are such that $\mathcal{L}>\eta$ and no clouds will form.

In case $\mathcal{L}(\eta) \leq \eta$, cloud formation will occur and an alternative equation for $h$ is needed, since $h \neq \eta$. In typical bulk models, the system is then closed using the assumption that $\mathcal{L}=\eta$. Much success has been achieved using this assumption, and we will start by using this closure as well.

It is worthwhile to investigate the premises for this closure. The equation for $\eta$ is based on the height that dry thermals reach, whereas $\mathcal{L}$ is the lifting condensation level following from $\theta_{l, m}$ and $q_{t, m}$. This difference between $\eta$ and $\mathcal{L}$ was already recognized by Betts and Ridgway (1989). Why would dry thermals be depleted of their energy exactly at the lifting condensation level?

Consider a situation where $\mathcal{L}(\eta)<\eta$; that is, $\eta-$ $\mathcal{L}(\eta)>0$. Thermals will now reach the lifting condensation level and form clouds from that level onward. The extra energy from the release of latent heat allows $h$ to increase beyond $\eta$. As a result, the thermodynamic properties of the layer are modified, which in turn lead to a modified lifting condensation level $\mathcal{L}^{\prime}=\mathcal{L}(h)$ and, in the most general case, to a modified $\eta^{\prime}$.

Two possibilities can be distinguished. If $\eta^{\prime}-\mathcal{L}^{\prime}<$ $\eta-\mathcal{L}$, the lifting condensation level approached the dry 
thermal reach. This allows fewer thermals to reach this level, thereby limiting the total latent heat release. In the extreme event that $\mathcal{L}^{\prime}>\eta^{\prime}$, no thermals will reach $\mathcal{L}^{\prime}$ and no latent heat release follows. This is a self-correcting mechanism with the classical closure as the limit:

$$
\mathcal{L}=\eta .
$$

As $\mathcal{L}$ is a function of $h$, Eq. (11) can be regarded an implicit equation for the steady-state boundary layer height $h$.

The second possibility, however, is that $\eta^{\prime}-\mathcal{L}^{\prime}>\eta-\mathcal{L}$. This would allow more thermals to reach lifting condensation level, increasing latent heat release. This increase would result in a further increased $h$, which in turn results in $\eta^{\prime \prime}-\mathcal{L}^{\prime \prime}>\eta^{\prime}-\mathcal{L}^{\prime}$, etc. This situation is unstable. It becomes apparent that to exclude such instability, we must require that

$$
\frac{\partial \eta-\mathcal{L}}{\partial h}<0
$$

at some height $h$ for a stable steady-state boundary layer with that height to be possible.

In conclusion, we need Eq. (12) as a premise to close the system using $\mathcal{L}=\eta$. Moreover, this closure is a steady-state result of the adaptation of the boundary layer to the thermodynamic effect of latent heat release. For that reason, the use of this closure in a timedependent framework implicitly assumes that the time scales of this feedback are much smaller than the other time scales in the system.

\section{c. Free atmosphere}

In the current framework we also assume the free troposphere to be in steady state, which entails that Eq. (2) also applies to $\bar{\psi}^{f}(z)$ for all $z>h$. Because the turbulent fluxes are zero for $z>h$, and because the equation must hold for any value of $h$, one arrives at the following relation between the source $S_{\psi}(z)$, the subsidence profile, and the free-tropospheric profile $\bar{\psi}^{f}(z)$ :

$$
\bar{w}(z) \frac{\partial \bar{\psi}^{f}(z)}{\partial z}=S_{\psi}(z) .
$$

The sources (advection, radiation, etc.) balance the effect of subsidence throughout the atmosphere, independent of $h$.

\section{d. Solutions}

Given $\psi \in\left\{\theta_{l}, q_{t}\right\}$, the steady-state solution can now be summarized as follows. Equation (5) can be solved for the mixed-layer values of $\theta_{l}$ and $q_{t}$. In our aim to simplify the model to its essence, we start by setting $\gamma=1$. This allows a smooth transition from cloudless to cloudy regimes. The result is

$$
\begin{aligned}
& \theta_{l}=\theta_{l}^{f}(h)-\frac{\phi_{\theta_{l}, 0}+\int_{0}^{h^{+}} S_{\theta_{l}} d z}{\bar{w}(h)} \text { and } \\
& q_{t}=q_{t}^{f}(h)-\frac{\phi_{q_{t}, 0}+\int_{0}^{h^{+}} S_{q_{t}} d z}{\bar{w}(h)} .
\end{aligned}
$$

Note that we have dropped the overbars as well as the subscript $m$ on $\left\{\theta_{l}, q_{t}\right\}$ for readability. Unless explicitly mentioned otherwise, hereafter $\theta_{l}$ and $q_{t}$ denote $\theta_{l, m}$ and $q_{t, m}$, respectively.

The dry thermal reach acts as a bound to determine whether a cloud layer appears. It can be solved from the implicit equation [Eq. (9)], repeated here in order to compactly present all model equations:

$$
-\phi_{\theta_{v l}, 0}(1+a)=\int_{0}^{\eta^{-}} S_{\theta_{v l}}(z) d z .
$$

Provided $\partial \mathcal{L} / \partial h>0, h$ can be solved from Eq. (11):

$$
h=\left\{\begin{array}{lll}
\eta & \text { if } & \mathcal{L}(\eta)>\eta \\
\mathcal{L}^{-1}(\eta) & \text { if } & \mathcal{L}(\eta) \leq \eta
\end{array}\right.
$$

In other words, if clouds form, the boundary layer height is found by solving $\mathcal{L}(h)=\eta$ for $h$. In the absence of clouds, $h=\eta$ and the solution reduces to the mixed-layer equations for the clear boundary layer, as formulated by Tennekes (1973) and still used in recent studies (VilàGuerau de Arellano et al. 2004; van Driel and Jonker 2011), when these are solved for steady state. In that case, the equation $d h / d t=w^{e}+\bar{w}(h)=0$ requires the entrainment rate $w^{e}$ to balance the subsidence, which is directly implied in Eqs. (14)-(16).

\section{Model settings}

\section{a. Case setup}

To describe the environment in a simplified yet generic way, we define the free-atmospheric state as the profile that the troposphere would have if there were no boundary layer (extrapolating downward). Now consider the following idealized free-atmospheric profiles (dashed lines in Fig. 2):

$$
\begin{aligned}
& \theta_{l}^{f}(z)=\theta_{l}^{f 0}+\Gamma z \quad \text { and } \\
& q_{t}^{f}(z)=q_{t}^{f 0} .
\end{aligned}
$$




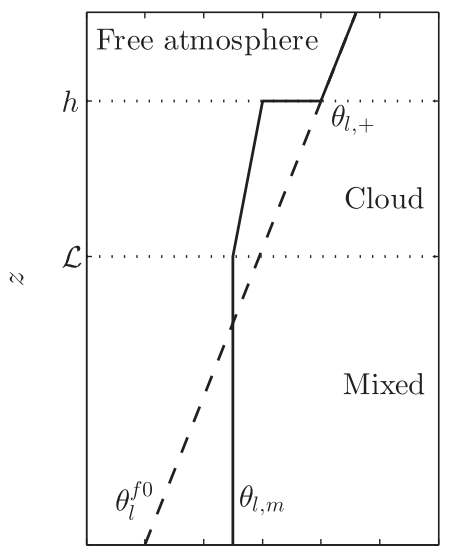

$\theta_{l}$

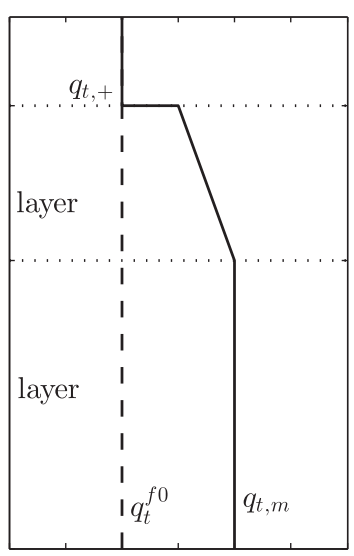

$q_{t}$
FIG. 2. Schematic picture of the connection between the idealized "free atmosphere" profile, extending from above the boundary layer all the way down to the ground (dashed lines), and the actual profile of the atmosphere, with a boundary layer up to $z=h$ (solid line). The boundary layer is divided into a well-mixed part and a cloudy part.

The lapse rate $\Gamma$ is kept constant over height and in time. For given $\bar{w}(z)$, Eqs. (18) and (19) also determine the sources through Eq. (13).

The formation of the boundary layer can alter these profiles only up to the $h$ such that a top boundary condition is automatically supplied. The resulting "jumps" $\Delta \psi=\psi_{+}-\psi_{m}=\psi^{f}(h)-\psi_{m}$ are defined by $\psi_{m}$ and $h$ as follows:

$$
\begin{aligned}
& \Delta \theta_{l}=\theta_{l}^{f 0}+\Gamma h-\theta_{l, m} \quad \text { and } \\
& \Delta q_{t}=q_{t}^{f 0}-q_{t, m} .
\end{aligned}
$$

A schematic picture of this notion is drawn in Fig. 2. The values of the parameters $\Gamma, \theta_{l}^{f 0}$, and $q_{t}^{f 0}$ are provided in Table 1. All large-eddy simulations performed in this study start with initial profiles as described by Eqs. (18) and (19); that is, $\theta_{l}(z)=\theta_{l}^{f}(z)$ and $q_{t}(z)=$ $q_{t}^{f}(z)$ at $t=0$.

By prescribing the free-atmospheric conditions and the subsidence profile (as we will), the sources are defined by Eq. (13); that is, $S_{q_{t}}=0$ and

$$
S_{\theta_{l}}=\bar{w} \Gamma \text {. }
$$

\section{b. Parameter evaluation}

The entrainment efficiency sets the maximum height that dry thermals reach and thus controls the boundary layer height of the cloudless boundary layer. Therefore,
TABLE 1. Environmental parameters used for the phase-space exploration.

\begin{tabular}{cl}
\hline \hline Parameter & \multicolumn{1}{c}{ Value } \\
\hline$\theta_{l}^{f 0}$ & $290 \mathrm{~K}$ \\
$q_{t}^{f 0}$ & $0 \mathrm{~kg} \mathrm{~kg}^{-1}$ \\
$\Gamma$ & $6 \times 10^{-3} \mathrm{~km}^{-1}$ \\
$p_{s}$ & $102900 \mathrm{~Pa}$ \\
\hline
\end{tabular}

we diagnose $a$ from all cloudless large-eddy simulations (15 in total) presented in sections $4 \mathrm{a}$ and $4 \mathrm{~b}$. The LES model is described in appendix A. The boundary layer height that the simulations reach is diagnosed, after which $a$ is chosen such that Eq. (16) best captures the trend.

On the basis of those data, we have found $a$ to be best described by a value of 0.4 . For this diagnosis, we defined $\eta$ as the location of the maximum gradient in the virtual potential temperature profile, following Sullivan et al. (1998). While this value for $a$ might seem large, it represents the best zero-order representation of the boundary layer profile in cloudless cases. It does not represent the minimum of the buoyancy flux, which will always be smaller in magnitude. Note that $a$ is the only control parameter in this zero-order model.

A short discussion on the numerical value of $a$ is provided in appendix B.

\section{c. Phase-space explorations}

In this study we will investigate the behavior of the boundary layer for different external forcings. In particular, we will consider the following cases.

Case I: In section 4a, the model is studied in case of a constant divergence $\mathcal{D}$; that is, $\bar{w}(z)=-\mathcal{D} z$. To begin, we explore the simple case of fixed surface fluxes, in which we study the influence of their magnitude and composition.

Case II: In section 4b, we explore the effect of coupled surface fluxes, varying the sea surface temperature and $\mathcal{D}$. The effect of the simplifying assumption to set $\gamma=1$ will be considered in section $4 \mathrm{c}$. We will investigate the influence of $\gamma$ and the physical mechanism it captures, reconsidering the results of section $4 \mathrm{~b}$.

Case III: In section $4 \mathrm{~d}$ the subsidence is chosen constant with height. Among others, this allows us to test the relevance of the assumption that $\partial \mathcal{L} / \partial h>0$, since $\partial \mathcal{L} / \partial h=0$ in this case.

Finally, in section 5 the dominant mechanisms will be identified and the behavior in different forcing regimes will be compared. 


\section{Results}

\section{a. Case I: Fixed fluxes and divergence}

To begin, we explore the simple case of fixed surface fluxes in case of constant divergence; that is, $\bar{w}(z)=-\mathcal{D} z$. In this case, Eqs. (13)-(16) can be evaluated as

$$
\begin{aligned}
& \theta_{l}(h)=\theta_{l}^{f 0}+\frac{\phi_{\theta_{l}, 0}}{\mathcal{D} h}+\frac{1}{2} \Gamma h \text { and } \\
& q_{t}(h)=q_{t}^{f 0}+\frac{\phi_{q_{t}, 0}}{\mathcal{D} h}
\end{aligned}
$$

with the dry thermal reach

$$
\eta=\left[\frac{2 \phi_{\theta_{v l}, 0}(1+a)}{\mathcal{D} \Gamma}\right]^{1 / 2} .
$$

Note that in this case, $\partial \eta / \partial h=0$. Now, the dependence of the lifting condensation level on $h$ can be investigated by expanding

$$
\frac{\partial \mathcal{L}}{\partial h}=\frac{\partial \mathcal{L}}{\partial q_{t}} \frac{\partial q_{t}}{\partial h}+\frac{\partial \mathcal{L}}{\partial \theta_{l}} \frac{\partial \theta_{l}}{\partial h}
$$

where we know that $\partial \mathcal{L} / \partial q_{t}<0$ and $\partial \mathcal{L} / \partial \theta_{l}>0$ from thermodynamical arguments. Therefore, substituting Eqs. (23) and (24) and using the fact that $h>\eta$, Eq. (26) can be used to show that

$$
\frac{\partial \mathcal{L}}{\partial h}>0
$$

for $\phi_{\theta_{v l}, 0}>0$. This justifies the use of Eq. (17), which together with Eqs. (23)-(25) describes the steady-state solution of $\left\{\theta_{l}, q_{t}, h, \mathcal{L}\right\}$ if the parameters $\left\{\phi_{\theta_{l}, 0}, \phi_{q_{t}, 0}\right.$, $\left.\theta_{l}^{f 0}, q_{t}^{f 0}, \Gamma, \mathcal{D}\right\}$ are provided.

To illustrate these solutions in a phase space of external parameters, we describe the surface fluxes in terms of the surface buoyancy flux $B_{0} \equiv \rho c_{p} \phi_{\theta_{v l}, 0}\left(\mathrm{~W} \mathrm{~m}^{-2}\right)$ and the Bowen ratio

$$
\beta=\frac{c_{p}}{L_{v}} \frac{\phi_{\theta_{l}, 0}}{\phi_{q_{t}, 0}},
$$

where $c_{p}$ is the specific heat capacity of water and $L_{v}$ is the latent heat of vaporization. The fluxes are prescribed and have a constant value over time.

The choice of surface fluxes allows a simple interpretation throughout the phase space. The surface buoyancy flux describes how much kinetic energy is brought into the boundary layer from the surface, and thereby governs $\eta$. Note that Eq. (25) is a function of the surface buoyancy flux only; so for a given surface buoyancy flux, $\eta$ is independent of the Bowen ratio.

The Bowen ratio determines how the surface energy is divided over latent and sensible heat. Therefore, it governs the values of $\theta_{l}$ and $q_{t}$ and thus the critical lifting condensation level $\mathcal{L}(\eta)$. A lower Bowen ratio corresponds to a moister and cooler boundary layer, while $\eta$ remains constant. This obviously corresponds to a situation more favorable to cloud formation.

The results are shown in Fig. 3. Figure 3a illustrates the model solution by depicting the cloud-layer depth $\zeta \equiv h-\mathcal{L}$ using shaded contours. The solutions are compared with a number of LESs. Each LES starts with initial profiles equal to the idealized free-atmospheric state described by Eqs. (18) and (19), thus without an initial boundary layer. Environmental parameters are provided in Table 1, and we prescribed a divergence of $\mathcal{D}=7 \times 10^{6} \mathrm{~s}^{-1}$. The LESs are further described in the appendix.

The simulations are performed for 12 days to allow the simulations to reach a steady state. Each symbol in Fig. 3a represents a separate LES, circles denote cases with $\zeta<50 \mathrm{~m}$, and squares denote cases with a steady $\zeta \geq 50 \mathrm{~m}$; their color indicates the cloud-layer depth. As we study the phase space in the close vicinity of the cloudless-cloudy boundary, LESs in the "cloudless" regime will still feature some especially moist updrafts that reach their condensation point. Therefore, we chose $\zeta=50 \mathrm{~m}$ as the boundary for LESs, as simulations with less than that will typically have a significantly lower liquid water path. The transition from clear to cloudy is illustrated more quantitatively in Fig. 3c.

Simulations which either ran into the top of the domain or did not reach steady state are depicted with a plus sign and are not considered in Figs. 3c-f. This only occurs for very thick cloud layers and is likely to be an artifact of the LES setup: reperforming random samples of such cases with a larger (vertical) domain and integrating over longer times, these simulations do reach well-defined steady states.

A simulation is considered to have reached a steady state if the 6-h-mean altitude $z_{\text {top }}$, defined as the lowest altitude where $q_{t}\left(z_{\text {top }}\right) \leq 1 \times 10^{-5} \mathrm{~kg} \mathrm{~kg}^{-1}$, remains steady over at least $24 \mathrm{~h}$.

Each LES ends in its own state, closely corresponding to the model predictions, while each started from the same initial conditions. Figure $3 b$ shows the time series of cloud depth for selected simulations with $B_{0}=$ $20 \mathrm{~W} \mathrm{~m}^{-2}$. This panel shows how cases with large Bowen ratios remain cloudless, while those with decreasing Bowen ratios develop deeper cloud layers, while each simulation started without clouds altogether. The simulation 

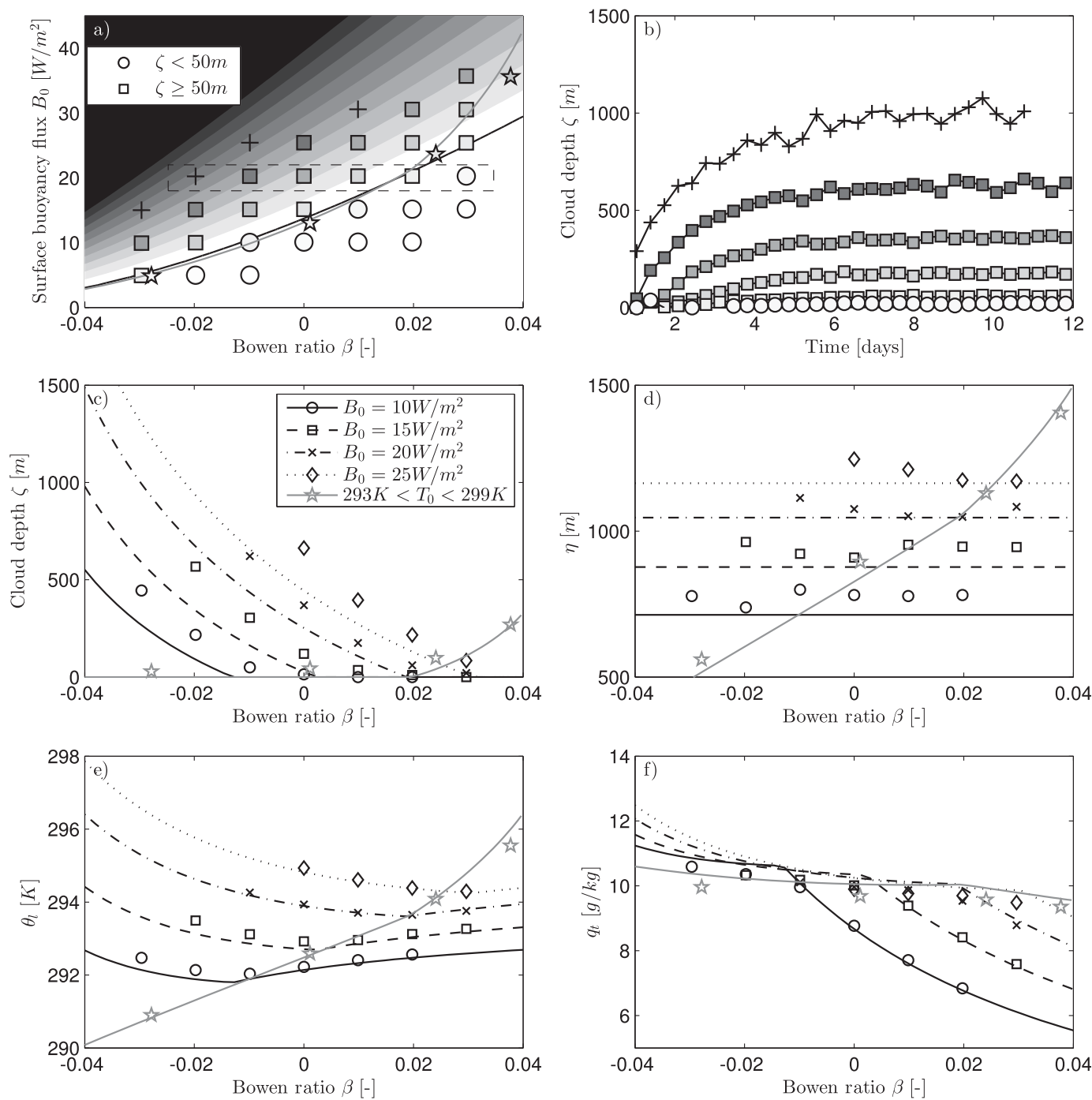

FIG. 3. Steady-state solutions for parameters as in Table 1 in a phase space of $B_{0}$ and $\beta$. (a) The shaded contours illustrate the qualitative behavior of the model prediction for $\zeta=h-\mathcal{L}$, with the black line representing the separation between cloudy and cloudless regimes. The overlaid symbols each depict the state of an LES after 12 days of simulated time, with the shape and fill color representing the LES's cloud-layer depth. (b) Time series of the cloudlayer depth for the case of $B_{0}=20 \mathrm{~W} \mathrm{~m}^{-2}$ are shown. (c)-(f) Model results for cloud-layer depth, mixed-layer height, and mixed-layer $\theta_{l}$ and $q_{t}$ are outlined in more detail through lines for selected values of $B_{0}$, while the symbols depict LES results. The gray lines illustrate the path that is traversed if fluxes are set interactively as a function of the sea surface temperature.

with $\beta=-0.02$ ran into the domain top, but the time series does suggest converging behavior.

Given the surface buoyancy flux, the model predicts a clear boundary layer at equal height for all large-enough Bowen ratios. Clouds form as the Bowen ratio decreases, increasing the boundary layer height. The mixed-layer values of $\theta_{l}$ and $q_{t}$ change accordingly, changing $\mathcal{L}$ up to the point that $\mathcal{L}=\eta$. Considering that $\eta$ in Eq. (25) is a function of surface buoyancy flux only, the surprising result is that the steady-state lifting condensation level is determined by the surface buoyancy flux, and independent of Bowen ratio.

This behavior is compared with LES results in more detail in Figs. 3c and 3d. Although the cloud-layer depth is systematically underestimated by the model, the trend is similar to LES. LESs with equal surface buoyancy flux show remarkably little variation in the mixed-layer height, which is diagnosed by considering the average cloud-base height or-in cloudless cases-the maximum gradient in the virtual potential temperature. 
The underestimation of cloud-layer depth is most likely to result from neglecting the cloud-layer gradient. A further discussion on this subject is provided in section $4 \mathrm{c}$.

Figures $3 \mathrm{e}$ and $3 \mathrm{f}$ show the mixed-layer values of the temperature and humidity. Especially in humidity, the effect of cloud formation is readily observed. For low Bowen ratios, the moistening of the mixed layer is inhibited by the fact that clouds form, which ventilates the excess moisture into the cloud layer (Neggers et al. 2006). Cloud formation simultaneously increases the entrainment of dry and warm air into the boundary layer, explaining the warming for low Bowen ratios in Fig. 3e.

\section{b. Case II: Fixed sea surface temperature}

One could argue that the approach of assuming constant surface fluxes is unrealistic, even in the stationary limit. For one, such an approach neglects the feedback that occurs as the result of the difference between the boundary layer state and the surface properties. An interesting approach, therefore, is to allow this feedback to occur by introducing limited surface-atmosphere interaction as follows:

$$
\begin{aligned}
& \phi_{\theta_{l}, 0}=V\left(\theta_{l, 0}-\theta_{l}\right) \quad \text { and } \\
& \phi_{q_{t}, 0}=V\left(q_{t, 0}-q_{t}\right)
\end{aligned}
$$

where $\theta_{l, 0}$ is the liquid water potential temperature related to the sea surface temperature $T_{0}$ via surface pressure $p_{s}$ and $q_{t, 0}$ the saturation humidity at $T_{0}$. In this approach, we have simplified the transfer coefficient $V=$ $C_{D}|U|$ as a constant to avoid feedbacks in the velocity $U$ and to allow for nonzero surface fluxes while remaining in the idealized case of no mean winds. We set $V$ to the value of $1 \mathrm{~cm} \mathrm{~s}^{-1}$, which would correspond to the transfer coefficient of a case with typical values of $U=5 \mathrm{~m} \mathrm{~s}^{-1}$ and $C_{D}=2 \times 10^{-3}$.

When this surface parameterization is introduced into Eqs. (14) and (15), the steady-state solutions become

$$
\begin{aligned}
& \theta_{l}(h)=\frac{\mathcal{D} h \theta_{l}^{f 0}+V \theta_{l, 0}+(1 / 2) \Gamma \mathcal{D} h^{2}}{\mathcal{D} h+V} \text { and } \\
& q_{t}(h)=\frac{\mathcal{D} h q_{t}^{f 0}+V q_{t, 0}}{\mathcal{D} h+V},
\end{aligned}
$$

revealing an explicit combination of surface and top conditions acting on the boundary layer. The surface buoyancy flux reaches a steady-state solution as follows:

$$
\phi_{\theta_{v l}, 0}(h)=\frac{\mathcal{D} h V}{\mathcal{D} h+V}\left(\theta_{v l, 0}-\theta_{v l}^{f 0}-\frac{1}{2} \Gamma h\right),
$$

where $\theta_{v l, 0}=\theta_{l, 0}+\widetilde{\epsilon}_{I} q_{t, 0}$ represents the sea surface virtual potential temperature.

The largest increase in complexity with regard to the previous section appears in the solution for the mixedlayer height. The solution for the mixed-layer height, Eq. (25), remains valid:

$$
\eta(h)=\left[\frac{2 \phi_{\theta_{v l}, 0}(h)(1+a)}{\mathcal{D} \Gamma}\right]^{1 / 2},
$$

but since the magnitude of the surface buoyancy flux is dependent on $h$, so is $\eta$. In case of cloudless convection, though, Eq. (34) is an implicit equation for $\eta$ since then $h=\eta$.

\section{PHASE SPACE}

The coupling between cloud layer and mixed layer has become further intertwined through air-surface interaction. Whereas $h$ already depended on the mixed-layer dynamics in case I, $\eta$ was independent of the boundary layer height until now. The result is that none of the model variables $\left\{\theta_{l}, q_{t}, \mathcal{L}, h\right\}$ can be regarded as independent of the other.

Nevertheless, when the steady state has been reached, fluxes are constant and as such, the system is equivalent to the system with constant surface fluxes. Essentially, we have lost a degree of freedom in the setup of the cases, since the surface fluxes are now governed by a single parameter (the sea surface temperature $T_{0}$ ) instead of two. Varying the sea surface temperature, with all other parameters held constant, thus amounts to following a trajectory through the $\left\{\beta, B_{0}\right\}$ phase space in Fig. 3. This path is visualized as a gray line in all panels of Fig. 3, representing the steady-state solution in case of an interactive surface flux found by varying $T_{0}$ from 293 to $299 \mathrm{~K}$. These solutions are compared to four large-eddy simulations shown by star-shaped markers. The line starts at $\beta=-0.03$ and $B_{0}=5 \mathrm{~W} \mathrm{~m}^{-2}$ at $T_{0}=293 \mathrm{~K}$. At this point evaporation drives the boundary layer to such an extent that the entrainment of warm air causes the boundary layer to become warmer than the sea surface, resulting in a negative sensible heat flux. Similar behavior was observed by Nuijens and Stevens (2012). As $T_{0}$ increases, $\beta$ and $B_{0}$ increase to about 0.03 and $30 \mathrm{~W} \mathrm{~m}^{-2}$, respectively.

For a fair comparison, the LES sea surface feedback was simplified to behave as Eqs. (29) and (30), with the mixed-layer values replaced by the first model level. The simulations are not found exactly on the gray line as they are free to develop surface fluxes that might differ from the model solutions. They are very close, however, demonstrating how an interactive flux mechanism moves through the surface flux phase space. Note that the 

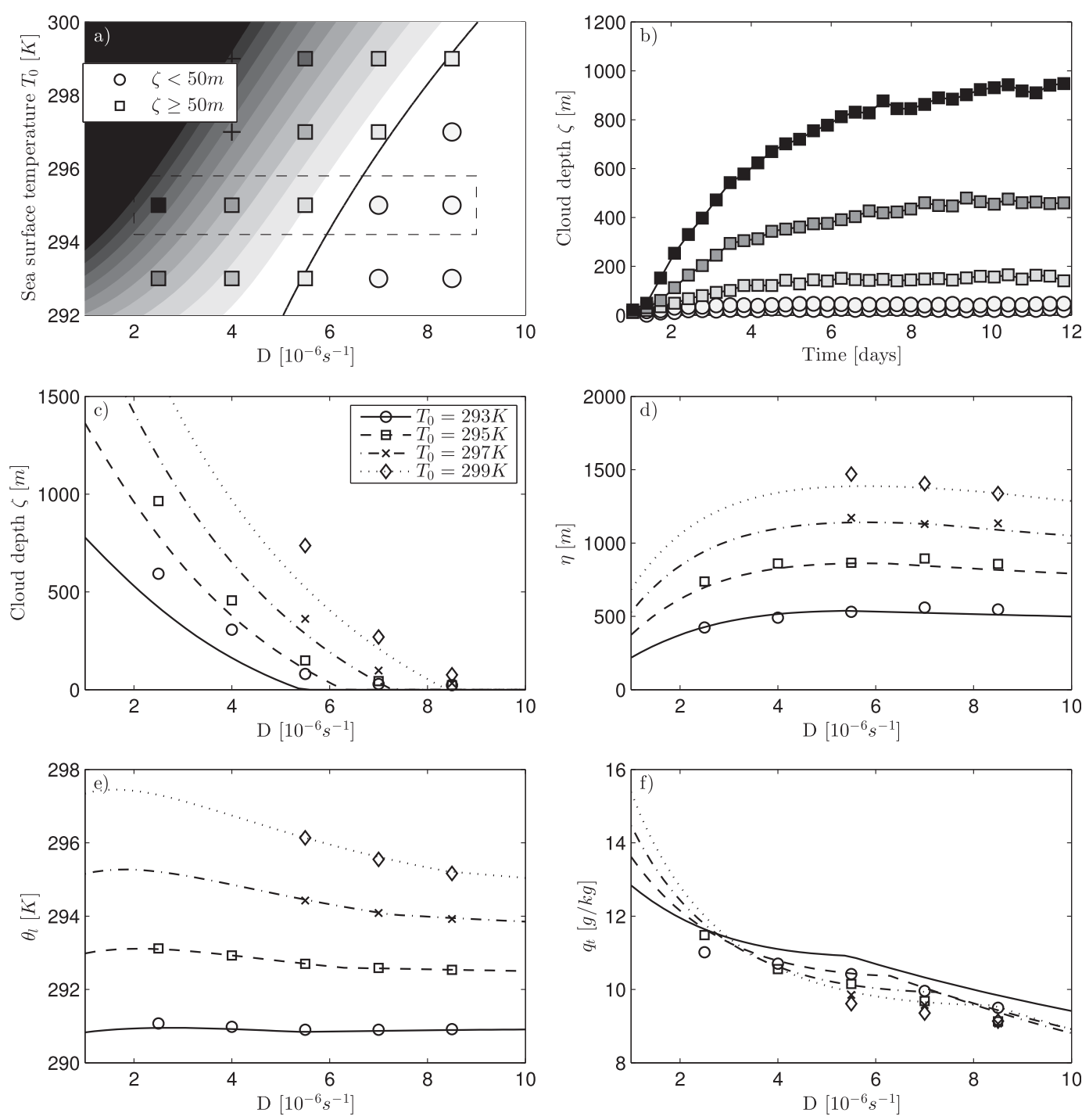

FIG. 4. Steady-state solutions for parameters as in Table 1. (a) Shaded contours depict the model for $\zeta=h-\mathcal{L}$, and overlaid symbols depict LESs as before. (b) Time series of the cloud-layer depth for $T_{0}=295 \mathrm{~K}$ are shown. (c)-(f) Model results for cloud-layer depth, mixed-layer height, and mixed-layer $\theta_{l}$ and $q_{t}$ are outlined in more detail in line plots for selected values of $T_{0}$.

surface fluxes traverse the phase space in a line nearly parallel to the boundary separating cloudy and cloudless regimes.

Figure $3 \mathrm{a}$ also illustrates the reason we chose to study the regime of rather low Bowen ratios, $-0.04 \leq \beta \leq 0.04$, since this is where the regime change in varying $T_{0}$ occurs. For comparison, the Barbados Oceanographic and Meteorological Experiment (BOMEX) intercomparison case (Siebesma et al. 2003) has a Bowen ratio of 0.06 (but has noncomparable free-tropospheric conditions).

Since we have lost a degree of freedom in the surface fluxes, we can now more easily study the behavior as a function of $\mathcal{D}$ as well. We have constructed a phase space of $T_{0}$ and $\mathcal{D}$ in Fig. 4 with equivalent panels as Fig. 3. The line through the surface flux phase space of Fig. 3 is now the vertical line in Fig. $4 \mathrm{a}$ at $\mathcal{D}=7 \times 10^{-6} \mathrm{~s}^{-1}$. The choice to scan a phase space of sea surface temperature and subsidence allows us to perform all LESs, like before, starting from the exact same initial conditions. The sea surface temperature and divergence are varied; the cooling by radiation and large-scale horizontal advection varies such that the divergence is always balanced [Eq. (13)]. This implies that the sink term increases in amplitude with $\mathcal{D}$; that is, the cooling is reduced if $\mathcal{D}$ decreases and vice versa.

Even though the added feedbacks increase the complexity in the dynamics of the system, the general behavior can be understood using the notions from previous 
sections. An increased sea surface temperature results in an increase in $B_{0}$ and $\beta$. Given constant free-atmospheric conditions, an increased sea surface temperature therefore leads to a warmer, moister, and therefore higher boundary layer. A warmer sea favors cloud formation as the increased buoyancy allows thermals to reach the lifting condensation level more easily.

At the same time, the dry thermal reach typically increases as divergence decreases, corresponding to intuition. Cumulus clouds, therefore, form more easily in regions of lower divergence, as this allows the mixed layer to deepen and reach the lifting condensation level.

Typical parameter values for the trade wind areas in the current climate (Bony et al. 2006; Zhang et al. 2009) correspond to the upper-left corner of Fig. 4a. It should be no surprise that this region is nested firmly in the cumulus regime.

Large-eddy simulations confirm the model predictions in the same fashion as they did in section 4a. Again, the general behavior is well reproduced. This is especially true for the mixed-layer height, which shows even better correspondence than in Fig. 3. As before, however, simulations tend to form deeper cloud layers than the model predicts.

We can conclude that the eventual state of the cloudy and cloudless boundary layer is, given the simplicity of the model, quite well described by four relatively simple equations [see Eqs. (14)-(17)].

\section{c. Cloud-layer effects}

So far we have ignored the cloud-layer gradients. Bellon and Stevens (2012) accounted for these effects by taking a constant value for $\gamma$ in Eq. (4) and representing the cloud-layer structure introducing $\alpha$ :

$$
\alpha=\frac{1}{h-\mathcal{L}} \int_{\mathcal{L}}^{h^{+}} \frac{\bar{\psi}(z)-\psi_{m}}{\bar{\psi}_{+}-\psi_{m}} d z
$$

which was taken constant as well. Unlike $\gamma, \alpha$ does not include effects of the subsidence $\bar{w}(z)$.

Whereas Bellon and Stevens (2012) studied a number of cases in which $\alpha=0.4$ and $\gamma=0.8$ accurately described the cloud layer, we found that in the spectrum of cases studied here, the use of a constant $\gamma<1$ deteriorates the model results with respect to $\gamma=1$. This is probably caused by taking both $\alpha$ and $\gamma$ constant at the same time. Indeed, substituting a constant divergence in Eq. (4) yields the relation

$$
\gamma= \begin{cases}1-\alpha\left(\frac{h-\mathcal{L}}{h}\right) & \text { for } h>\mathcal{L} \\ 1 & \text { otherwise }\end{cases}
$$

where the dependence on $\mathcal{L}$ and $h$ directly reveals that $\alpha$ and $\gamma$ cannot both be constant.

To remedy this conflict, we included $\gamma$ using Eq. (36) and constant $\alpha$ (as $\alpha$ most directly describes the profile, whereas $\gamma$ is dependent on $\bar{w}$ ). The numerical value of $\alpha=0.3$ provided the best results. We can now solve Eq. (5) as before, but including the varying $\gamma(h, \mathcal{L})$. If we do this for the phase space described in section 4b, Fig. 5 results. Comparing Figs. 4 and 5, one difference directly stands out: the modeled cloudlayer depth-Figs. 5a and 5c-is significantly improved in the revised model. Most of the other model results, however, are remarkably similar. For instance, compare the mixed-layer parameters $\eta, \theta_{l}$, and $q_{t}$ of Figs. 5d-f.

The differences and similarities can be understood from the role of $\gamma$. First, in the cloudless limit $\gamma=1$ and the model reduces to the simpler model. In cloudy cases, however, the system adapts the mixed-layer parameters to the point that $\mathcal{L}=\eta$ [Eq. (11)]. This severely limits the possible variation in the mixed layer and explains much of the similarities.

What the cloud-layer gradients effectively do is to moderate the function $\mathcal{L}(h)$ such that more heat $\left(\theta_{l}\right)$ and less moisture is stored in the cloud layer. This requires the boundary layer to grow higher than it would in the absence of these gradients and explains the improved prediction of the cloud-layer depth.

However, some difficulty lies in the fact that $\gamma$ is dependent on the subsidence profile. As it is unrealistic to assume the magnitude of the subsidence to increase indefinitely, Bellon and Stevens (2012) consider an exponentially saturating subsidence profile. In the next subsection, we consider what happens in the high-altitude limit: a constant subsidence profile.

\section{d. Case III: Constant subsidence}

The high-altitude limit of an exponentially saturating subsidence profile, $\bar{w}=-w_{s}\left[1-\exp \left(z / z_{w}\right)\right]$, accounts to constant subsidence with height: $\bar{w}=-w_{s}$. Note that $\partial \bar{w} / \partial z$ enters the equations through $\gamma$ [Eq. (4)], which only acts on the cloud-layer profile $(\mathcal{L}<z<h)$. Hence, a constant subsidence profile can be interpreted as representing the limit of $z_{w} \ll \mathcal{L}$; that is, the profile saturates within the mixed layer. An interesting consequence of constant subsidence is that its introduction in Eq. (4) results in $\gamma=1$ irrespective of cloud-layer gradients. Hence, our assumption of the earlier sections becomes a requirement in this case.

To keep the equations as simple as possible, we return to the case of fixed fluxes. Equations (14)-(16) can then be evaluated for constant subsidence $\bar{w}(z)=-w_{s}$ as follows: 

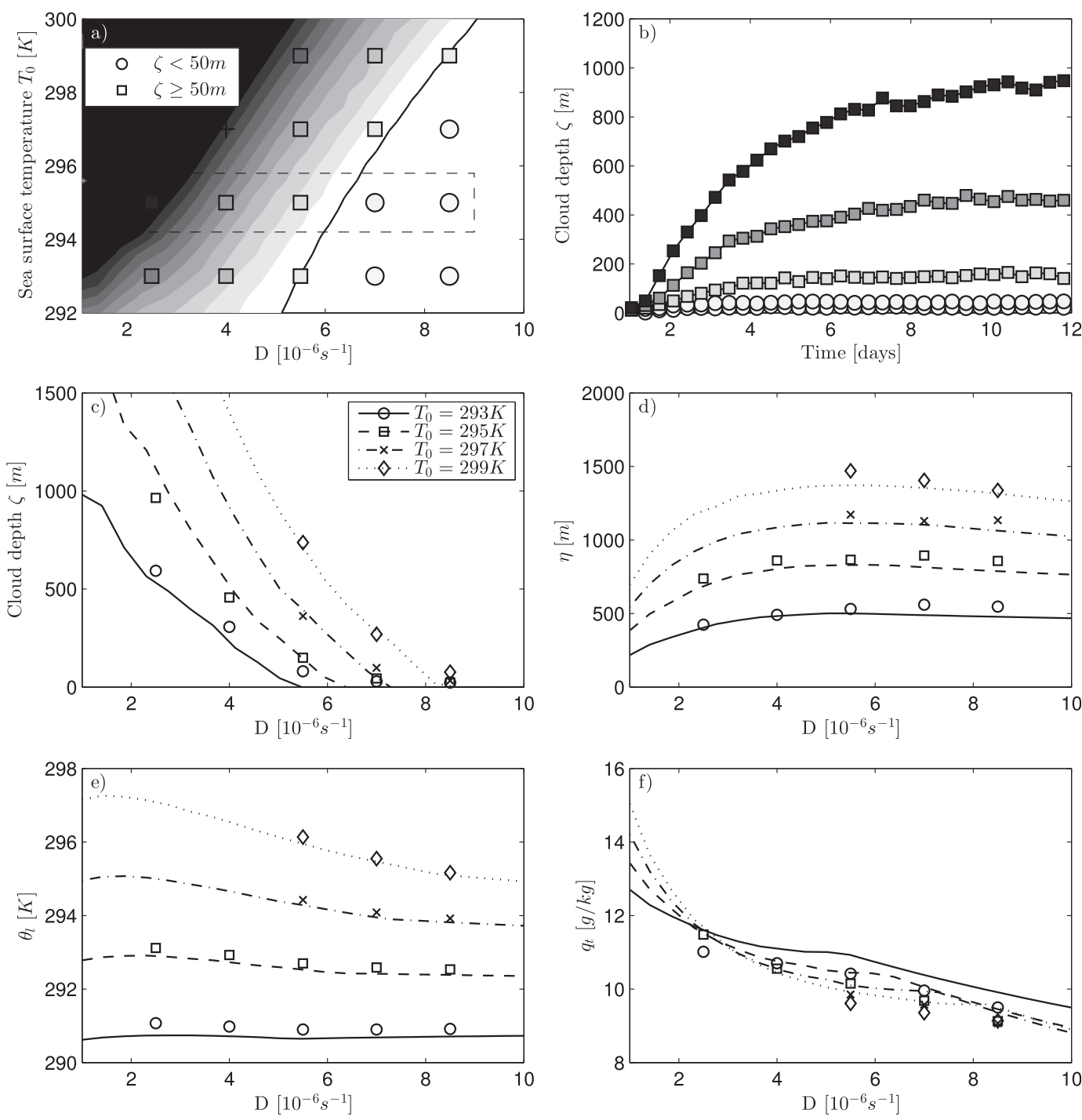

FIG. 5. Steady-state solutions for parameters as in Table 1. The depicted phase space and LES results are as in Fig. 4, but the model now includes a cloud-layer gradient through a variable value of $\gamma$ taken from Eq. (36) with $\alpha=0.3$.

$$
\begin{aligned}
& \theta_{l}=\theta_{l}^{f 0}+\frac{\phi_{\theta_{l}, 0}}{w^{s}} \text { and } \\
& q_{t}=q_{t}^{f 0}+\frac{\phi_{q_{t}, 0}}{w^{s}},
\end{aligned}
$$

with dry thermal reach

$$
\eta=\frac{\phi_{\theta_{v l}, 0}(1+a)}{w^{S} \Gamma},
$$

which renders the system similar to the one studied by van Driel and Jonker (2011).

There is an important difference between the system of Eqs. (37)-(39) as compared to those in case of constant divergence, Eqs. (23) and (24). In the current set of equations, $\theta_{l}$ and $q_{t}$ are independent of $h$. The result is that the lifting condensation level cannot adapt itself to the boundary layer dynamics; that is,

$$
\frac{\partial \eta}{\partial h}=0 \quad \text { and } \quad \frac{\partial \mathcal{L}}{\partial h}=0,
$$

which invalidates Eq. (17). This occurs as a result of the constant subsidence profile. Differentiating Eq. (5) with respect to $h$, we find

$$
\bar{w}(h) \frac{\partial \psi_{m}}{\partial h}=\underbrace{\bar{w}(h) \frac{\partial \bar{\psi}_{+}}{\partial h}-S_{\psi}(h)}_{0}+\frac{\partial \bar{w}(h)}{\partial h}\left(\bar{\psi}_{+}-\psi_{m}\right) .
$$



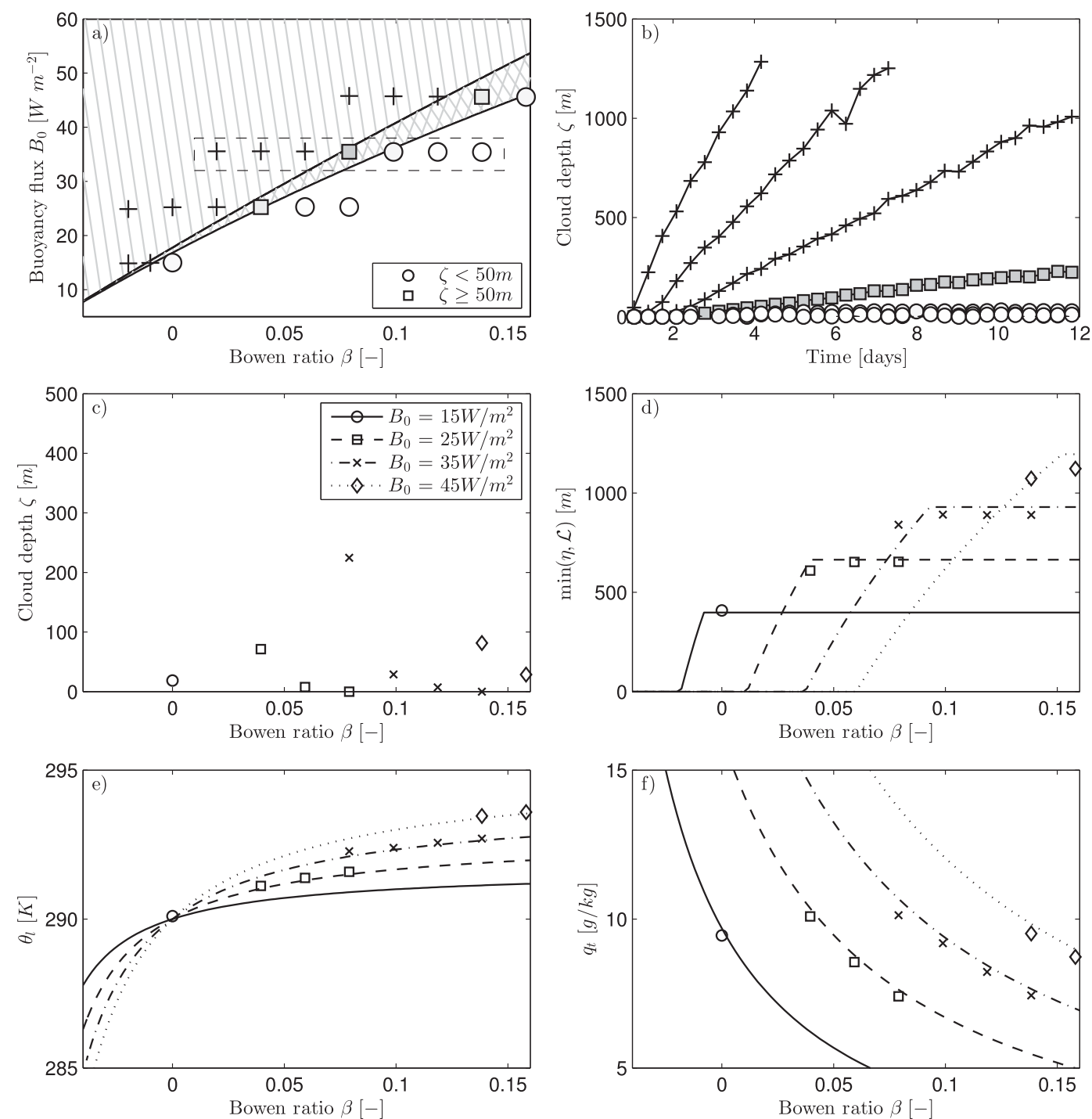

FIG. 6. Steady-state solutions for parameters from Table 1 as a function of $B_{0}$ and $\beta$. The hatched contours indicate regions of $\eta / \mathcal{L}$; no model solution for $\zeta$ is available. The overlaid symbols each depict the state of an LES after 12 days of simulated time, with the shape and fill color representing the LES's cloud-layer depth. (b) Time series of the cloudlayer depth for the case of $B_{0}=35 \mathrm{~W} \mathrm{~m}^{-2}$ are shown. (c)-(f) Model results for cloud-layer depth, mixed-layer height, and mixed-layer $\theta_{l}$ and $q_{t}$ are outlined in more detail in line plots for selected values of $B_{0}$.

The first two terms on the right-hand side cancel because of the stationarity of the free troposphere, Eq. (13). In case of constant subsidence, then, it is clear that $\psi_{m}$ must become independent of boundary layer height. Physically, the warming due to warmer entrained air for higher $h$ is exactly balanced by the sources (e.g., radiative cooling) the boundary layer now experiences.

This does not occur in the case of constant divergence, as the divergence introduces the extra nonzero term $\mathcal{D}\left(\bar{\psi}_{+}-\psi_{m}\right)$ on the right-hand side of Eq. (41). This results from the fact that the boundary layer has to entrain at an increased rate to balance the subsidence at level $h$.

\section{1) Phase space}

As the premise for the closure of Eq. (17) is not met, the model does not provide a prediction for $\zeta$. We evaluate the phase space as before but refrain from adding cloud-layer depth predictions in Fig. 6. Instead, we have added hatching for certain regimes of $\eta / \mathcal{L}$.

The lifting condensation level is a result of the mixedlayer values of $\theta_{l}$ and $q_{t}$, and is independent of $h$ in this case. Therefore, the feedback mechanism described in section $2 \mathrm{~b}$ has become dysfunctional, and $\mathcal{L} \neq \eta$ in general. The physical distinction between $\mathcal{L}$ and $\eta$ is now inescapable. 
By comparing $\mathcal{L}$ and $\eta$, three different regimes can be distinguished which show distinctly different behavior.

\section{2) REGIME I: $\mathcal{L}>\eta$-CLEAR LAYER}

In the first regime, filled white in Fig. 6a, the lifting condensation level is found above the mixed-layer height in steady state. No clouds form, because rising air will not reach its condensation level before reaching the inversion. A clear boundary layer will develop with a boundary layer height $h=\eta$.

3) REGIME II: $\eta /(1+a)<\mathcal{L}<\eta$

If the steady-state values $\theta_{l}$ and $q_{t}$ change, $\mathcal{L}$ changes accordingly. If $\mathcal{L}$ is found below $\eta$, condensation occurs within the boundary layer and clouds form.

We limit this regime such that that the minimum buoyancy flux remains below zero:

$$
\phi_{\theta_{v}}^{\min } \leq 0 \quad \text { for } \quad \frac{\mathcal{L}}{\eta} \geq \frac{1}{1+a},
$$

which follows from integrating Eq. (7) up to $\eta /(1+a)$, assuming the minimum buoyancy flux is found just below $\mathcal{L}$.

This regime, cross hatched in Fig. 6a, is defined such that the corresponding buoyancy flux reaches negative values, requiring thermals to reach their condensation level through inertia: a path has to be traveled in which they are negatively buoyant. This allows only the stronger thermals to condensate, as is typical for cumulus clouds. This regime therefore spans the range at which steadystate cumulus clouds are expected to be found.

4) REGIME III: $\mathcal{L}<\eta /(1+a)$-COUPLED REGIME

This leads us to consider the regime where the minimum buoyancy flux fails to become negative:

$$
\phi_{\theta_{v}}^{\min }>0 \quad \text { for } \quad \frac{\mathcal{L}}{\eta}<\frac{1}{1+a} ;
$$

see the hatched area in Fig. 6a.

Observations suggest (de Roode and Duynkerke 1997) that the degree of coupling is important in regulating the amount of moisture transport into the cloud layer. Among other reasons, this causes the cloud-base (minimum) buoyancy flux to be considered an important parameter when considering the regime boundary between cumulus and stratocumulus (Bretherton and Wyant 1997; Stevens 2000). A negative cloud-base buoyancy flux then typically implies decoupling of the mixed layer and the cloud layer, usually resulting in a cumulus layer.

In the current regime, where the environment is moist enough for the minimum buoyancy flux to remain positive, the mixed layer is expected to couple with the cloud layer. The amount of moisture ventilated into the cloud layer is too much for the layer to stabilize and an unstable, ever-growing boundary layer results. Indeed, the time series in this regime [Eq. (6b)] show no signs of convergence.

\section{5) MOdel PERFORMANCE}

The behavior of the LES closely resembles the model predictions for $\eta$ and mixed-layer values of $\theta_{l}$ and $q_{t}$ as before. Although the model is incapable of predicting a steady-state boundary layer height at this point, it seems the general regimes are well represented. The LES time series in Fig. 6b clearly indicate the instability of the cases in regime III, the behavior being markedly different from that in case of constant divergence (Fig. 3b).

The ever-increasing boundary layer height in this regime results in an increasing liquid water content. At some point, longwave radiative cooling of the cloud layer will no longer be negligible, such that the present model framework breaks down. Longwave radiative cooling might account for stabilization in this regime, and might induce a regime change to stratocumulus. Whereas it might be a future possibility to expand the model with a stratocumulus-entrainment formulation (e.g., Nicholls and Turton 1986; Moeng 2000), further investigation of the coupled layer state remains outside the scope of this paper.

Also note that the stability of regime II might seem to conflict with the fact that $(\partial \eta-\mathcal{L}) / \partial h=0$, which is inconsistent with Eq. (12). However, Eq. (12) followed from linear arguments: the case of constant subsidence is neither linearly stable nor linearly unstable. Therefore, higher-order terms control the stability, likely causing the interesting physical behavior.

\section{Synthesis}

The behavior of the cloud layer varies quite significantly over the different cases in the previous section, motivating an overview of the similarities and differences.

\section{a. Constant subsidence}

The advantage of studying cases under a subsidence profile constant with height is that steady-state solutions are independent of boundary layer height. This is due to the fact that in steady state, entrainment always balances subsidence, implying that steady-state entrainment is independent of height. Combined with the fact that the free troposphere is in equilibrium, the differences in entrainment with increasing $h$ are balanced by source terms (e.g., radiation). 
This causes the mixed-layer thermodynamical properties to become independent of the cloud layer, and hence so does the lifting condensation level. The result is that cumulus clouds have become something close to a passive property of the boundary layer. We can now identify one of the main preconditions of a steady-state cumulus layer: the lifting condensation level has to have a height such that thermals from the surface have energy enough to reach it, $\mathcal{L}<\eta$, yet also such that the minimum buoyancy flux is smaller than zero, $\mathcal{L}>\eta /(1+a)$.

\section{b. Divergence}

By introducing a subsidence profile that increases in height because of $\mathcal{D}$, the boundary layer is allowed an extra mechanism. Through increasing the cloud-layer depth, the boundary layer has to entrain at a faster rate to balance the increased subsidence. The result is a boundary layer that adapts its cumulus cloud-layer depth to find a height with just the right thermodynamical properties for cumulus to persist.

The cumulus-capped boundary layer in this case is stable as a perturbation that decreases $h$ results in a decrease in $\mathcal{L}$. This increases latent heat release, which increases the buoyancy of rising thermals and thus $h$, stabilizing the solution. LES evidence of unstable solutions in case of constant subsidence, where $\mathcal{L}$ is independent of $h$, strengthens this conclusion.

\section{c. Sea-air interaction}

The introduction of sea-air interaction, irrespective of subsidence, alters the steady-state solutions in no fundamental way. It does clarify the relative influence of the surface and top (free atmosphere) as it constrains the values of surface buoyancy flux and Bowen ratio.

Overall, cumulus clouds develop and increase in height as the sea surface temperature increases and divergence (or subsidence) decreases. This is in good accordance with the ubiquity of shallow cumulus in the trade wind regimes (e.g., Stevens 2006; Rauber et al. 2007).

\section{d. Cloud-layer gradients}

The effect of cloud-layer gradients is to further increase the cloud-layer depth. The mechanism in which the lifting condensation level must balance the reach of dry thermals severely restricts the possible variation in mixedlayer properties. The effect of cloud-layer gradients in this mechanism is to alter the distribution of $\theta_{l}$ and $q_{t}$ over height in such a way that the lifting condensation level reacts less strongly to an increased boundary layer height. The result is that the boundary layer height-and hence the cloud-layer depth—can increase farther than it could without gradients.

\section{e. Comparison}

The governing mechanism in cumulus development seems to be the adaptation of $\mathcal{L}$ to $\eta$. To unify the results of previous sections, let us introduce the dry thermal reach for the dry boundary layer as $\eta_{0}$, the height the boundary layer would reach if condensation is ignored. In case of prescribed sea surface temperature, $\eta_{0}=$ $f\left[\phi_{\theta_{v l}, 0}\left(\eta_{0}\right)\right]$; in all other cases, $\phi_{\theta_{v l}, 0}$ is independent of $h$ and therefore $\eta_{0}=\eta$.

The hypothetical lifting condensation level corresponding to a boundary layer with height $h=\eta_{0}$ is $\mathcal{L}_{0}=$ $\mathcal{L}\left(\eta_{0}\right)$. This allows the various phase spaces of previous sections to be combined into a single phase space of $\mathcal{L}_{0}$, $\eta_{0}$, shown in Fig. 7. Note that in essence, $\mathcal{L}_{0}$ and $\eta_{0}$ are found by solving the classical mixed-layer model of the $\mathrm{CBL}$, and then calculating $\mathcal{L}_{0}$ from the mixed-layer values of $\theta_{l}$ and $q_{t}$ a posteriori.

The cloud-formation threshold is then described by the $\mathcal{L}_{0}=\eta_{0}$ line, as clouds never form for larger $\mathcal{L}_{0}$. Lower $\mathcal{L}_{0}$ require a more intense adaptation in order to reach the balance $\mathcal{L}=\eta$, and therefore correspond to thicker cloud layers. In case $\partial \bar{w} / \partial z=0$, the mixed layer cannot adapt $\mathcal{L}$ to meet $\eta$. This renders the solutions unstable for low $\mathcal{L}_{0} / \eta_{0}$ as the self-correcting feedback is now dysfunctional.

The similarity of all panels in Fig. 7 is striking, which shows how the dry thermal reach analysis unifies the different regimes.

\section{Discussion and concluding remarks}

Not many of the concepts described in this work are new in themselves. In fact, the modeling framework is for a large part based on the mixed-layer modeling approach formulated by Tennekes (1973). Although less often applied to cumulus convection than to the clear boundary layer, cumulus modeling was already shown possible by Lilly (1968) and Betts (1973), and many followed since then. The explicit distinction between lifting condensation level and dry thermal reach in the equations originates from Betts and Ridgway (1989), even though it has been used only scarcely since.

The novelty in this work may lie in the "bulk" interpretation of the cumulus layer. Much work is available that describes cumulus cloud processes in detail and even models it accordingly. However, building upon bulk models of, most notably, Stevens (2007) and Bellon and Stevens (2012), our model treats the cloud layer as a whole as an integral part of the bulk boundary layer. We show that the role of the cloud-layer gradients in the qualitative behavior of the steady-state solutions is limited, allowing further simplification of bulk models 

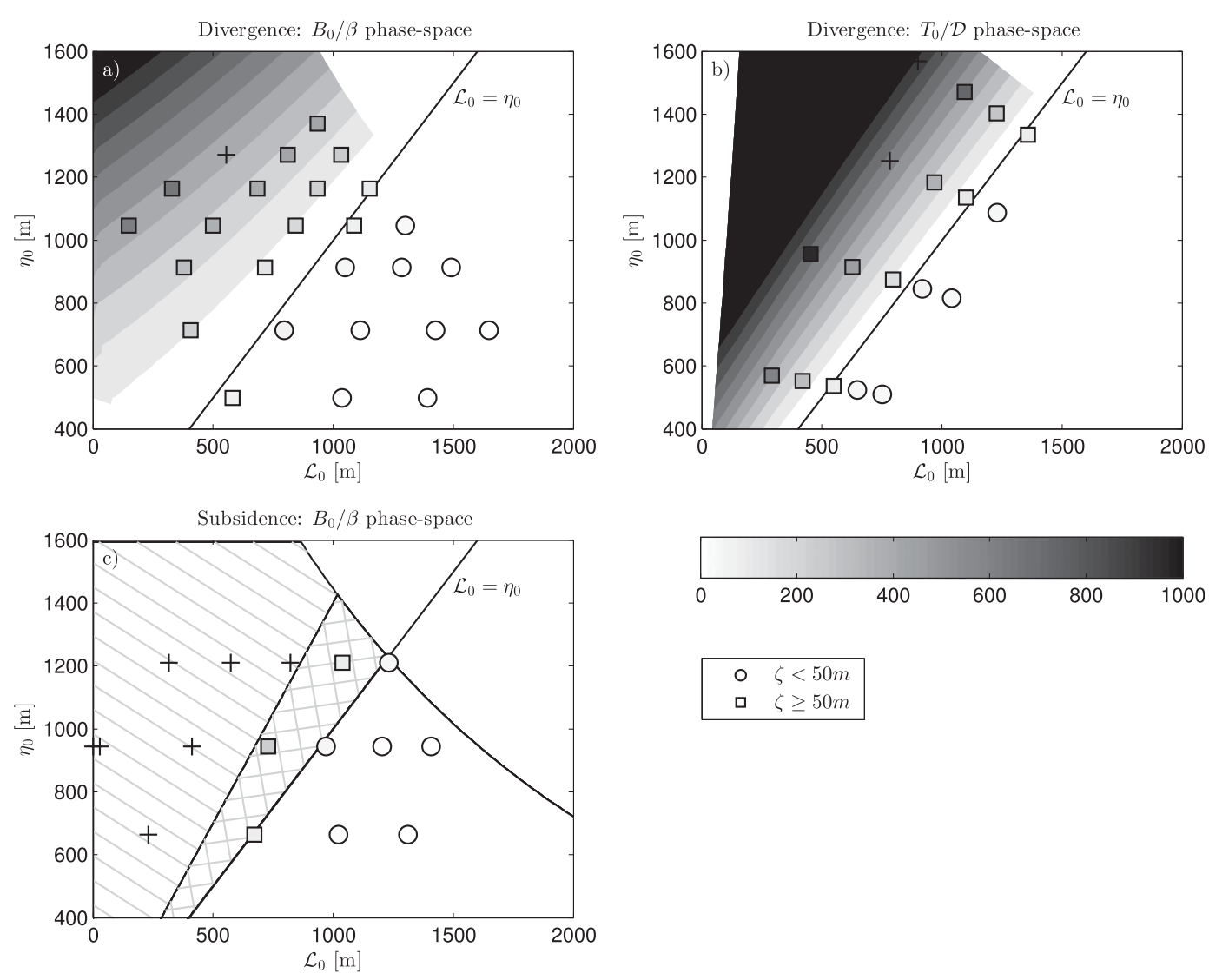

FIG. 7. Steady-state solutions for parameters from Table 1, varying (a) surface fluxes for $\mathcal{D}=7 \times 10^{-6} \mathrm{~s}^{-1}$, (b) $T_{0}$ and $\mathcal{D}$, and (c) surface fluxes for $w_{s}=7 \mathrm{~mm} \mathrm{~s}^{-1}$. Model results are compared with large-eddy simulations, whose shape and fill color represent the cloud-layer depth. The diagonal lines represent $\mathcal{L}_{0}=\eta_{0}$.

without losing the ability to interpret the dominant processes.

The model suggests that the cloud-layer depth is not determined by how much latent heat individual cloudy thermals have and how high this allows them to penetrate into the free atmosphere, even though this governs the dynamics of an individual cloud. Instead, the reach of dry thermals sets a benchmark to which the wet thermals can adapt. This explains the performance of the classical closure of the dry entrainment efficiency beneath the cloud, irrespective of the fundamentally different origin of this process, noted by Betts and Ridgway (1989).

Second, the presented equations readily describe the steady-state regimes. This further simplifies the process of finding cumulus regimes, since a numerical solution to the presented equations is enough, abandoning the need for model integration in time.

Combined with the fact that this framework allows for such a solution without describing processes that are important to the internal cloud structure, such as the mass flux development and lateral entrainment and detrainment, this could aid the setup of numerical experiments of steady-state cumulus in varying conditions. Such experiments can be a further aid in studying the response of these process-level cloud-layer properties to varying temperature, humidity, and forcings.

In this way, the present framework can also be informative on the problem of low-cloud feedback. Through applying realistic changes in SST and divergence, in line with climate model projections under a doubled- $\mathrm{CO}_{2}$ scenario, the modified steady-state solutions provide information on the sign and strength of the feedback in the trade wind cumulus regime (Zhang and Bretherton 2008).

Of course, in reality the boundary layer has to react to environmental forcings that hardly resemble the idealized settings used in this study. Nevertheless, we hope to have identified and even isolated some of the processes through which a cumulus layer influences the boundary layer system in response to external forcings, providing many avenues for further studies. 
Acknowledgments. The authors thank Dr. G. Bellon and two anonymous reviewers for their useful comments. The work is part of the European Union Cloud Intercomparison, Process Study and Evaluation project (EUCLIPSE) of the Seventh Framework Programme (FP7/20072013) and received partial funding under Grant Agreement 244067. This work was sponsored by the National Computing Facilities Foundation (NCF) for the use of supercomputer facilities, with financial support of NWO.

\section{APPENDIX A}

\section{LES Case Description}

The model results were assessed using a number of large-eddy simulations (LESs). Four categories of model results are presented in this paper, combining constant subsidence or constant divergence with constant fluxes or a constant sea surface temperature. Within each category, all simulations are performed with equal forcings and conditions as described in the relevant section.

Large-eddy simulations can be used to simulate the boundary layer processes in detail, while the forcings and initial profiles can be arbitrarily set. For this research, all simulations have been performed using the graphics processing unit (GPU)-resident Atmospheric LargeEddy Simulation (GALES) (Schalkwijk et al. 2012) of the Dutch Atmospheric Large-Eddy Simulation described in Heus et al. (2010). Using GPU acceleration allows the simulations to be performed locally, such that new simulations can be quickly set up and run with different forcings. GALES can also use the GPU to visualize the cloud field during the simulation itself. The resulting graphical representation of the present clouds provides rapid feedback on the current boundary layer state. The large speedup in "time-to-solution" ratios increases the viability of long-time-scale (i.e., steady state) simulations.

All simulations are performed on a domain of $6.4 \mathrm{~km}$ in both horizontal directions and $3.06 \mathrm{~km}$ in the vertical direction. Grid spacing is $24 \mathrm{~m}$ in the vertical direction and $50 \mathrm{~m}$ in the horizontal directions. Initial conditions are described by Eqs. (18) and (19) for all categories, with values tabulated in Table 1 . The simulations are performed with constant prescribed surface fluxes for $\theta_{l}$ and $q_{t}$ for the relevant categories, or with a simplified surface scheme for a constant sea surface temperature, described by Eqs. (29) and (30). In the latter case, the transfer coefficient $V$ is constant and prescribed, and the temperature and humidity differences are those between the surface and first model level.

The forcings are described by constant (in time) and prescribed radiative cooling and subsidence profiles,
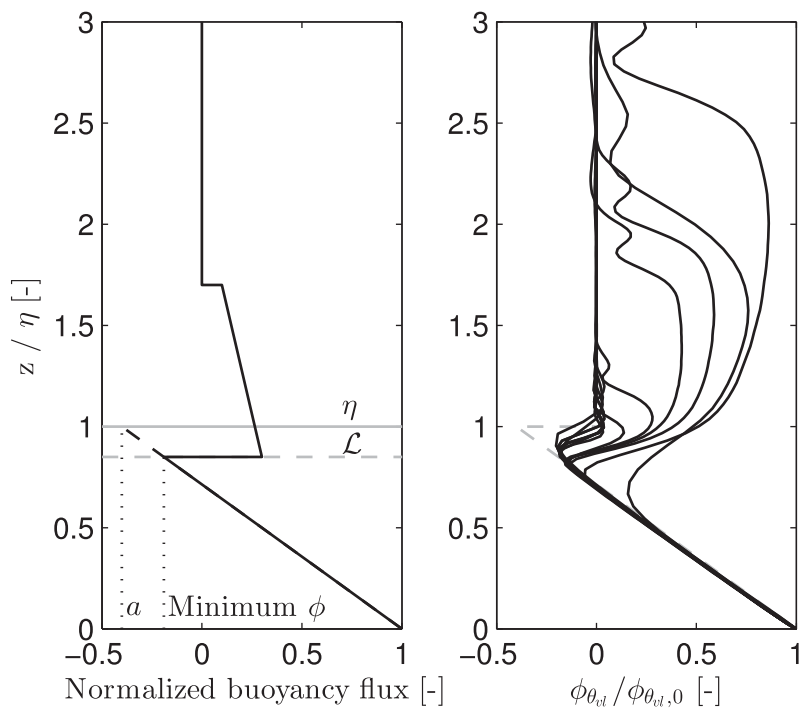

FIG. B1. (left) Schematic picture of the relation between the buoyancy flux and $\eta$ as well as $\mathcal{L}$. (right) Actual buoyancy fluxes of all simulations used in Fig. 6, averaged over the last $10 \mathrm{~h}$ of the simulation (i.e., after 12 days).

either constant in height or continually increasing with height. The large-scale subsidence advects scalars downward without influencing momentum in order to avoid violating the incompressibility condition in a periodic domain (Heus et al. 2010). No geostrophic wind is present, in accordance with the model framework. Equally, no interactive or cloud-dependent radiation is applied in the large-eddy simulations such as to remain consistent with the model.

\section{APPENDIX B}

\section{Entrainment Efficiency}

Since the value of the entrainment efficiency $a$ used in this study is relatively large ( 0.4 versus the typical $0.2-$ $0.25)$, Fig. B1 shows the mechanism behind the numerical value. The left panel illustrates how $a$ and $\eta$ are intrinsically coupled in their definition. We determine $a$ such that Eq. (16) provides a good prediction for $h=\eta$ in cloudless cases. Effectively, this boils down to extrapolating the flux profile up to the point at which Eq. (8) is met.

Compare the normalized LES profiles in the right panel with the illustration in the left panel. The fact that all flux profiles in the mixed layer collapse on a single line after normalization with $\eta$ and $\phi_{\theta_{v l}, 0}$ is a strong argument for the choice of $a$. Note that in cloudless cases (where the flux profile exhibits no positive excursion above the mixed layer), the turbulent fluxes vanish at $h=\eta$ as assumed in section 2 . 
As expected, if $\mathcal{L}<\eta$ (which is possible in steady state for the case of constant subsidence depicted here), the actual profile will depart from the mixed-layer slope from $z=\mathcal{L}$ up. In case $\mathcal{L}>\eta$, the profile will depart from the mixed-layer slope because of the "entrainment zone," which is neglected in a zero-order approximation. Therefore, the minimum value of the buoyancy flux is always smaller in magnitude than $-a \phi_{\theta_{v l}, 0}$

Finally, note that the value of $a$ used in this study was chosen to best match our LES model at the given resolution. Different LES models might result in slightly different values of $a$, as might varying resolutions. The conceptual model framework, however, remains similar. As such, $a$ can be regarded the single "tuning" parameter of the model. The reader is referred to van Driel and Jonker (2011) for a further discussion on the value of the entrainment efficiency in mixed-layer models, including the dependence on resolution.

\section{REFERENCES}

Ball, F., 1960: Control of inversion height by surface heating. Quart. J. Roy. Meteor. Soc., 86, 483-494.

Bellon, G., and B. Stevens, 2012: Using the sensitivity of large-eddy simulations to evaluate atmospheric boundary layer models. J. Atmos. Sci., 69, 1582-1601.

$\longrightarrow$, and -2013 : Time scales of the trade wind boundary layer adjustment. J. Atmos. Sci., 70, 1071-1083.

Betts, A., 1973: Non-precipitating cumulus convection and its parameterization. Quart. J. Roy. Meteor. Soc., 99, 178-196.

—, and W. Ridgway, 1989: Climatic equilibrium of the atmospheric convective boundary layer over a tropical ocean. J. Atmos. Sci., 46, 2621-2641.

Bony, S., and Coauthors, 2006: How well do we understand and evaluate climate change feedback processes? J. Climate, 19, 3445-3482.

Bretherton, C., and M. Wyant, 1997: Moisture transport, lowertropospheric stability, and decoupling of cloud-topped boundary layers. J. Atmos. Sci., 54, 148-167.

— implications for penetrative entrainment feedback on updraft buoyancy. J. Atmos. Sci., 65, 2174-2193.

Deardorff, J. W., 1970: Preliminary results from numerical integrations of the unstable planetary boundary layer. J. Atmos. Sci., 27, 1209-1210.

de Roode, S., and P. Duynkerke, 1997: Observed Lagrangian transition of stratocumulus into cumulus during ASTEX: Mean state and turbulence structure. J. Atmos. Sci., 54, $2157-$ 2173.

Dufresne, J., and S. Bony, 2008: An assessment of the primary sources of spread of global warming estimates from coupled atmosphere-ocean models. J. Climate, 21, 5135-5144.

Grenier, H., and C. S. Bretherton, 2001: A moist PBL parameterization for large-scale models and its application to subtropical cloud-topped marine boundary layers. Mon. Wea. Rev., 129, 357-377.

Heus, T., and H. Jonker, 2008: Subsiding shells around shallow cumulus clouds. J. Atmos. Sci., 65, 1003-1018.
- and Coauthors, 2010: Formulation of the Dutch Atmospheric Large-Eddy Simulation (DALES) and overview of its applications. Geosci. Model Dev., 3, 415-444.

Lilly, D., 1968: Models of cloud-topped mixed layers under a strong inversion. Quart. J. Roy. Meteor. Soc., 94, 292-309.

Moeng, C., 2000: Entrainment rate, cloud fraction, and liquid water path of PBL stratocumulus clouds. J. Atmos. Sci., 57, 36273643.

Neggers, R., B. Stevens, and J. Neelin, 2006: A simple equilibrium model for shallow-cumulus-topped mixed layers. Theor. Comput. Fluid Dyn., 20, 305-322.

Nicholls, S., and J. Turton, 1986: An observational study of the structure of stratiform cloud sheets: Part II. Entrainment. Quart. J. Roy. Meteor. Soc., 112, 461-480.

Nuijens, L., and B. Stevens, 2012: The influence of wind speed on shallow marine cumulus convection. J. Atmos. Sci., 69, 168-184.

Paluch, I., 1979: The entrainment mechanism in Colorado cumuli. J. Atmos. Sci., 36, 2467-2478.

Rauber, R., and Coauthors, 2007: Rain in shallow cumulus over the ocean: The RICO campaign. Bull. Amer. Meteor. Soc., 88, 1912-1928.

Reuter, G., and M. Yau, 1987: Mixing mechanisms in cumulus congestus clouds. Part I: Observations. J. Atmos. Sci., 44, 781797.

Schalkwijk, J., E. Griffith, H. Post, and H. Jonker, 2012: High performance simulations of turbulent clouds on a desktop PC: Exploiting the GPU. Bull. Amer. Meteor. Soc., 93, 307314.

Siebesma, A., and J. Cuijpers, 1995: Evaluation of parametric assumptions for shallow cumulus convection. J. Atmos. Sci., 52, 650-666.

— , and Coauthors, 2003: A large eddy simulation intercomparison study of shallow cumulus convection. J. Atmos. Sci., 60, 1201-1219.

Stevens, B., 2000: Cloud transitions and decoupling in shear-free stratocumulus-topped boundary layers. Geophys. Res. Lett., 27, 2557-2560.

_ 2006: Bulk boundary-layer concepts for simplified models of tropical dynamics. Theor. Comput. Fluid Dyn., 20, 279-304.

,- 2007 : On the growth of layers of nonprecipitating cumulus convection. J. Atmos. Sci., 64, 2916-2931.

Sullivan, P., C. Moeng, B. Stevens, D. Lenschow, and S. Mayor, 1998: Structure of the entrainment zone capping the convective atmospheric boundary layer. J. Atmos. Sci., 55, 3042-3064.

Tennekes, H., 1973: A model for the dynamics of the inversion above a convective boundary layer. J. Atmos. Sci., 30, 558-567.

van Driel, R., and H. Jonker, 2011: Convective boundary layers driven by nonstationary surface heat fluxes. J. Atmos. Sci., 68 , $727-738$.

Vilà-Guerau de Arellano, J., B. Gioli, F. Miglietta, H. J. J. Jonker, H. K. Baltink, R. W. A. Hutjes, and A. A. M. Holtslag, 2004: Entrainment process of carbon dioxide in the atmospheric boundary layer. J. Geophys. Res., 109, D18110, doi:10.1029/ 2004JD004725

Zhang, M., and C. Bretherton, 2008: Mechanisms of low cloudclimate feedback in idealized single-column simulations with the Community Atmospheric Model, version 3 (CAM3) J. Climate, 21, 4859-4878.

Zhang, Y., B. Stevens, B. Medeiros, and M. Ghil, 2009: Low-cloud fraction, lower-tropospheric stability, and large-scale divergence. J. Climate, 22, 4827-4844. 